\title{
Periprocedural Cost-Effectiveness Analysis of Mechanical Thrombectomy for Acute Ischemic Stroke in the Stent Retriever Era
}

\author{
Tareq Kass-Hout ${ }^{a} \quad$ Omar Kass-Hout ${ }^{a}$ Chung-Huan Sun ${ }^{a}$ Taha Kass-Hout ${ }^{c}$ \\ Samir R. Belagaje ${ }^{a}$ Aaron M. Anderson ${ }^{a} \quad$ Michael R. Frankel ${ }^{a}$ Rishi Gupta ${ }^{a}$ b \\ Raul G. Nogueira ${ }^{a}$ \\ a Department of Neurology, Emory University School of Medicine, Atlanta, Ga., \\ ${ }^{b}$ Neurosurgery, WellStar Medical Group, Marietta, Ga., and ' Humanitarian Tracker, Vienna, Va., USA
}

\section{Key Words}

Acute ischemic stroke $\cdot$ Stent retriever $\cdot$ Cost-effectiveness $\cdot$ Tissue plasminogen activator

\begin{abstract}
Background: Early reperfusion is critical for favorable outcomes in acute ischemic stroke (AIS). Stent retrievers lead to faster and more complete reperfusion than previous technologies. Our aim is to compare the cost-effectiveness of stent retrievers to the previous mechanical thrombectomy devices. Methods: Retrospective review of endovascularly treated largevessel AIS. Data from all consecutive patients who underwent thrombectomy from January 2012 through November 2012 were collected. Baseline characteristics, the total procedural cost, the rates of successful recanalization [modified thrombolysis in cerebral ischemia (mTICI) scores of $2 \mathrm{~b}$ or 3$]$, and the length of stay at the hospital were compared between the stent retriever (SR) and the non-stent retriever (NSR) groups. Results: After excluding the patients who underwent concomitant extracranial stenting $(n=22)$ or received intra-arterial tissue plasminogen activator only $(n=6)$, the entire cohort included 150 patients. The cost of the reperfusion procedure was significantly higher in the SR compared to the NSR group (USD 13,419 vs. 9,308, $p<0.001$ ). We were unable to demonstrate a statistically significant difference in the rates of $\mathrm{mTICI} 2 \mathrm{~b} / 3$ reperfusion ( 81 vs. $74 \%, p=0.337$ ) or the length of stay (11.1 \pm 9.1 vs. $12.8 \pm 9.6$ days, $p=0.260$ ) amongst the SR and the NSR patients. Conclusion: The procedural costs of thrombectomy for AIS are increasing and account for the bulk of hospitalization reimbursement. The impact of these expenditures in the long-term sustainability of stroke centers deserves greater consideration. While it is likely that the SR technology results in higher rates of optimal reperfusion, better clinical outcomes, and shorter lengths of stay, larger studies are needed to prove its cost-effectiveness.




\section{Introduction}

Stroke is one of the most costly health problems in America and the Western world [1, 2], with estimated direct and indirect costs of USD 38.6 billion annually [3]. A pooled analysis of the ATLANTIS, ECASS, and NINDS rt-PA stroke trials demonstrated that the time from symptom onset to treatment is an independent predictor of favorable outcome after intravenous (IV) thrombolysis [4]. Time-dependent reperfusion has also been demonstrated with endovascular treatment of acute ischemic stroke (AIS) [5, 6]. The emergence of stent retrievers (SRs; the Solitaire ${ }^{\mathrm{TM}}$ Flow Restoration and the Trevo $^{\mathrm{TM}}$ Retriever devices) has helped to achieve more efficient reperfusion. Two randomized clinical trials have shown that these devices are significantly better in restoring blood flow in occluded arteries as compared to the MERCI Retriever [7, 8]. Consequently, the US Food and Drug Administration recently cleared both devices for clot retrieval in AIS patients. The increases in costs related to this novel 'more effective' technology may be justifiable if patients have better outcomes and may be financially neutral or even cost-saving if there is a reduction in hospital length of stay (LOS). The goal of this study is to compare the cost-effectiveness of SRs relative to the previous technologies (MERCI device or Penumbra aspiration system) in treating large-artery AIS.

\section{Methods}

Patient Selection and Outcome Measures

After approval from our institutional review board, we conducted a retrospective review of consecutive patients treated with endovascular therapy for AIS at the Marcus Stroke and Neuroscience Center at Grady Memorial Hospital from January 2012 through November 2012. SRs became the primary device utilized for thrombectomy on April 15, 2012. Patients who received extracranial stenting as part of the stroke intervention and patients who received intra-arterial tissue plasminogen activator (tPA) as the only stroke intervention were excluded from the final analysis. Our study cohort was then divided into two groups. The nonstent retriever (NSR) group consisted of patients who underwent mechanical thrombectomy with either the MERCI retrieval system (Stryker Neurovascular, Mountain View, Calif., USA) or the Penumbra system (Penumbra Inc., Alameda, Calif., USA) as the primary thrombectomy device. The SR group consisted of patients who received mechanical thrombectomy with either the Solitaire FR Revascularization Device (Covidien, Plymouth, Minn., USA) or the Trevo Pro Retrieval System (Stryker, Kalamazoo, Mich., USA) as the primary thrombectomy device.

The primary endpoint for this analysis was the total procedural cost. Values were calculated based on the list prices for the thrombectomy devices, microcatheter/microwire systems, access catheters and wires, and support catheters and/or balloon guide catheters whenever these were used. Items such as syringes, tubing, and contrast media as well as the costs related to personnel and the angiographic equipment were not considered in the analysis. However, the average cost of each good clinical outcome [modified Rankin Scale (mRS) 0-2] was not captured in our retrospective analysis. The secondary endpoints included the LOS in days in the neuroscience intensive care unit, the procedure time (defined as time from groin puncture to final angiographic image), the rate of successful near-complete or complete perfusion [defined as modified thrombolysis in cerebral ischemia (mTICI) scores of $2 \mathrm{~b}$ or 3 , e.g. reperfusion of $\geq 50 \%$ of the occluded territory], and the rate of good functional outcome (defined as mRS scores of 0-2 at 90 days after the procedure).

Statistics

IBM SPSS software version 20 was utilized to perform the desired statistical analysis. A simple twosample $t$ test was performed to compare continuous variables that were normally distributed. The MannWhitney U test was performed when comparing continuous variables that were not normally distributed. The Fisher exact test was used to compare categorical variables. Variables with $\mathrm{p}<0.20$ in the univariate analysis were then included in the binary logistic regression model-building process. The model was built using forward/backward stepwise logistic regression with variables entered into the model at the 0.05 significance level and removed at the 0.10 significance level. All analyses were performed by a biostatistician with the aid of STATA software (Stata ${ }^{\circledR} 13$ ). 
Table 1. Demographic and clinical characteristics

\begin{tabular}{lccc}
\hline Characteristic & SR $(\mathrm{n}=85)$ & NSR $(\mathrm{n}=65)$ & $\mathrm{p}$ value \\
\hline Age, years & $67.5 \pm 14.5$ & $63.1 \pm 15.5$ & 0.072 \\
Male gender & $47(55)$ & $32(49)$ & 0.461 \\
Hypertension & $67(79)$ & $52(80)$ & 0.860 \\
Diabetes mellitus & $20(24)$ & $20(31)$ & 0.320 \\
Dyslipidemia & $26(31)$ & $17(26)$ & 0.552 \\
Current smoker & $21(25)$ & $12(18)$ & 0.365 \\
NIHSS upon admission & $18.5 \pm 6.7$ & $18.4 \pm 5.6$ & 0.881 \\
Atrial fibrillation & $34(40)$ & $25(38)$ & 0.848 \\
LKN to groin puncture, min & $257 \pm 66.63$ & $258 \pm 64.43$ & 0.926 \\
\hline
\end{tabular}

Values are presented as mean \pm SD or $\mathrm{n}(\%)$.

NIHSS = National Institutes of Health Stroke Scale; LKN = last known normal.

\section{Results}

\section{Clinical and Treatment Characteristics}

A total of 179 consecutive patients underwent AIS intervention during the study period. Of those, 29 patients were excluded (22 patients received cervical carotid stenting, 6 patients received intra-arterial tPA only as an acute intervention, and 1 patient did not require mechanical thrombectomy due to spontaneous recanalization) to yield 150 patients for analysis. The mean age was $65.3 \pm 15$ years, the median National Institutes of Health Stroke Scale was $18.5 \pm 6.2$, and $52 \%$ were men. The site of occlusion included the middle cerebral artery in 83 patients (55.3\%), the internal carotid artery terminus in 52 patients $(34.6 \%$; one of these patients had a tandem occlusion of the middle cerebral artery), and the basilar artery in 15 patients $(10 \%)$.

Among the whole cohort, 85 (57\%) patients were in the SR group and 65 (43\%) patients were in the NSR group. Time from last seen normal to groin puncture was similar between the two groups ( $257 \pm 66.63 \mathrm{~min}$ SR vs. $258 \pm 64.43 \mathrm{~min}$ NSR, $p=0.926)$. Table 1 shows that the two groups were matched across the rest of the baseline variables except that patients in the NSR group tended to be younger compared to the SR group ( $p=0.072$ ).

\section{Primary and Secondary Clinical Outcome}

At the time of the analysis, only $94 \%(142 / 150 ; 79$ SR, 63 NSR) of the patients had their 90-day functional assessment. The remaining 8 patients were lost in the follow-up period. Table 2 summarizes the cost differences and outcomes in the two groups. The procedural cost of endovascular thrombectomy was significantly higher if an SR was used (USD 13,419 \pm 5,711 SR vs. 9,308 $\pm 3,848$ NSR, $p<0.001$ ). Notably, the devices' procedural cost alone for the SR and NSR treatments, respectively, comprised 44 and 31\% of the national Diagnosis Related Group that is used by Medicare to reimburse stroke centers for the cost of the total stay of stroke patients undergoing mechanical thrombectomy (Medicare Severity Diagnosis Related Group 024 = USD 30,197) [9]. The SR technology was associated with significantly higher rates of complete reperfusion (mTICI 3: 35\% SR vs. 10\% NSR, p < 0.001); however, there were no significant differences in the rates of near complete/complete reperfusion (mTICI $2 \mathrm{~b} / 3$ : $81 \%$ SR vs. $74 \%$ NSR, $p=0.337$ ) or good clinical outcomes (90-day mRS 0-2: 38\% SR vs. $36 \%$ NSR, $\mathrm{p}=0.847$ ) (table 2; fig. 1).

Similarly, the total hospitalization LOS and the LOS in the neuroscience intensive care unit were not different between the two groups $(11.1 \pm 9.1$ SR vs. $12.8 \pm 9.6 \mathrm{NSR}, \mathrm{p}=0.260$, 
Table 2. Procedural cost and patient outcome

\begin{tabular}{lccr}
\hline Outcome & SR $(\mathrm{n}=85)$ & NSR $(\mathrm{n}=65)$ & $\mathrm{p}$ value \\
\hline Cost, USD & $13,419 \pm 5,711$ & $9,308 \pm 3,848$ & $<0.001$ \\
Good outcome at 90 days (mRS $\leq 2)$ & $27 / 79(34)$ & $22 / 58(35)$ & 0.926 \\
Complete reperfusion (mTICI 3) & $30(35)$ & $6(10)$ & $<0.001$ \\
Near-complete reperfusion (mTICI 2b/3) & $69(81)$ & $47(74)$ & 0.337 \\
Total LOS, days & $11.1 \pm 9.1$ & $12.8 \pm 9.6$ & 0.260 \\
Length of ICU stay, days & $5.1 \pm 5.4$ & $5.2 \pm 4.9$ & 0.908 \\
PEG placement & $12(14)$ & $14(22)$ & 0.234 \\
Tracheostomy placement & $5(6)$ & $5(8)$ & 0.660 \\
Procedure time, min & $74.5 \pm 53.5$ & $78.4 \pm 42.2$ & 0.636 \\
\hline
\end{tabular}

Values are presented as mean \pm SD or n (\%).

ICU = Intensive care unit; PEG = percutaneous endoscopic gastrostomy.

Fig. 1. Schematic plots comparing the costs, reperfusion rate (mTICI $2 \mathrm{~b} / 3$ ), and good functional outcome (mRS 0-2) between the SR and NSR groups. ICU = Intensive care unit; PEG = percutaneous endoscopic gastrostomy.

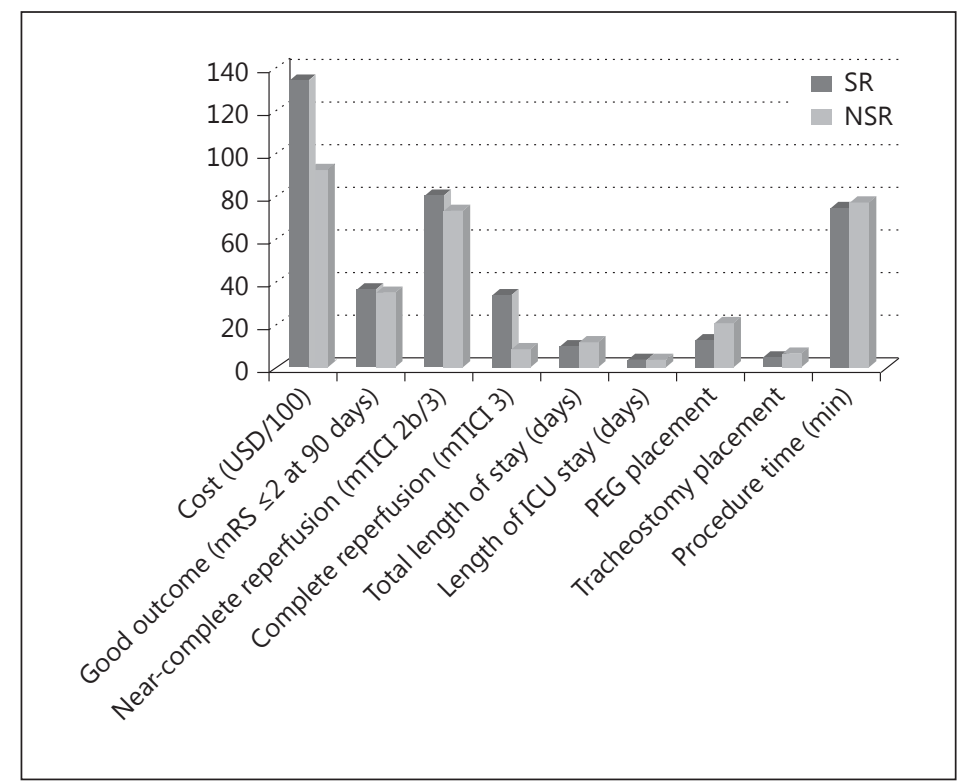

and $5.1 \pm 5.4$ SR vs. $5.2 \pm 4.9$ NSR, p = 0.908, respectively). Other clinical outcome measures, such as percutaneous endoscopic gastrostomy and tracheostomy placement, were also not different between the two groups ( $14 \%$ SR vs. $22 \%$ NSR, $p=0.234$, and $6 \%$ SR vs. $8 \%$ NSR, $\mathrm{p}=0.660$, respectively). Surprisingly, the procedure time (groin puncture to reperfusion) was not meaningfully shorter when SRs were used $(74.5 \pm 53.5 \mathrm{~min}$ SR vs. $78.4 \pm 42.2 \mathrm{~min}$ NSR, $\mathrm{p}=0.636$ ) (table 2).

\section{Multivariate Analysis of Predictors of Good Functional Outcome}

In view of the presence of interrelationships among all predictors of variables associated with good clinical outcome, a multiple logistic regression analysis was used and revealed the presence of 4 independent predictors of good functional outcome 90 days after acute stroke intervention: final infarct volume [odds ratio (OR), 0.97; 95\% confidence interval (CI), $0.95-$ 0.98; $\mathrm{p}<0.0001$ ], age (OR, 0.93; 95\% CI, 0.90-0.97; $\mathrm{p}=0.001$ ), LOS (OR, 0.86; 95\% CI, 0.79-0.94; $\mathrm{p}=0.001$ ), and glucose level on admission (OR, 0.98; 95\% CI, 0.97-0.99; $\mathrm{p}=0.007$ ) (table 3). 


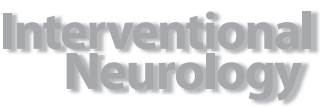

Table 3. Multivariate predictors of good functional outcome at 90 days after acute stroke intervention

\begin{tabular}{l|l}
\hline Intervent Neurol 2014;3:107-113 \\
\hline DOI: $10.1159 / 000371729$ & $\begin{array}{l}\text { C } 2015 \text { S. Karger AG, Basel } \\
\text { www.karger.com/ine }\end{array}$ \\
\hline
\end{tabular}

Kass-Hout et al.: Periprocedural Cost-Effectiveness Analysis of Mechanical Thrombectomy for Acute Ischemic Stroke in the Stent Retriever Era

\begin{tabular}{llc}
\hline Variable & OR $(95 \% \mathrm{CI})$ & $\mathrm{p}$ value \\
\hline Final infarct volume & $0.97(0.95-0.98)$ & $<0.0001$ \\
Age & $0.93(0.90-0.97)$ & 0.001 \\
LOS & $0.86(0.79-0.94)$ & 0.001 \\
Glucose level on admission & $0.98(0.97-0.99)$ & 0.007 \\
\hline
\end{tabular}

\section{Discussion}

Our initial data suggest that, despite the noteworthy rise of the procedural cost compared with the older-generation mechanical thrombectomy devices, the SR technology did not yield a higher rate of mTICI $2 b / 3$ reperfusion. The demonstration of an impact on LOS and functional outcomes may require a larger sample size. As one of the most common causes of death, long-term morbidity and disability, stroke causes a substantial economic burden. The aggregate lifetime cost of stroke in the first 2 years accounted for $45 \%$ of total costs, longterm ambulatory care accounted for $35.0 \%$, and nursing home costs accounted for $17.5 \%$, implying a robust correlation between severity of disability and direct care cost [10].

Good outcome after AIS depends on multiple factors, but early reperfusion plays a significantrole [11]. IV thrombolysis with recombinant tPA is currently the only approved treatment and has been shown to be cost-effective for patients with stroke by reducing health expenditures by $90 \%$ over 30 years [12]. However, the rates of partial or complete recanalization in large-vessel occlusions with IV tPA alone have been shown to be as low as $6 \%$ for terminal internal carotid artery occlusions [13]. Additionally, nonrecanalized acute intracranial largevessel occlusion was found to be an independent predictor of poor neurological outcome in $85 \%$ of patients [14]. These limitations had led to the emergence of iterative mechanical thrombectomy devices to achieve better revascularization.

On the basis of the available data, mechanical therapies in qualified patients with acute stroke beyond the window for IV tPA [15] and the combination of IV TPA and mechanical thrombectomy for large-vessel ischemic stroke appears to be cost-effective [16]. Even though mechanical thrombectomy is usually offered later in the course of acute stroke when revascularization is generally less effective, a sensitivity analysis based on the results of the MERCI study [17] showed that, although mechanical thrombectomy carries an approximately USD 6,600 higher cost compared with standard medical therapy, it remained cost-effective [18]. The substantially improved recanalization rate with the Solitaire FR Revascularization Device and the Trevo Pro Retrieval System compared to the MERCI Retriever [7, 8] has changed clinical practice with interventionists increasingly adopting newer-generation technologies. In comparison with a previous report suggesting that aspiration appears to be the most costeffective method to achieve acceptable recanalization rates with low complication rates [19], our study suggests that, even though treatment with SRs was approximately USD 4,000 more expensive than that with the older mechanical thrombectomy devices, it yields significantly higher rates of mTICI 3 reperfusion. Future analyses with larger sample sizes are required to determine the impact on clinical outcomes, LOS, and other procedural costs such as percutaneous endoscopic gastrostomy tube placement. It remains possible that the extra cost associated with the use of SR will be easily overcome by these other expenditures as well as by the improved functionality and greater productivity of the treated patients. Indeed, a prospective European study suggested that any reduction of the disability level will dramatically reduce the cost burden, as the costs of care of a stroke patient whose mRS score is 4 or 5 was three times the cost of care of a patient with an mRS score of $0-2$, and it was $70 \%$ higher 
than for a patient with an mRS score of 3 [20]. Another study illustrated that patients with symptomatic stroke would trade 10 years of life with their disability for 6.6 years of life without disability [21]. Based on these data, neurothrombectomy utilizing SR appears to still be a cost-effective intervention compared with older-generation thrombectomy devices. This cost-effectiveness estimate should be reassessed once data from randomized controlled trials of mechanical thrombectomy become available. Our study has significant limitations including its retrospective and relatively small sample size, but it brings into question important aspects of stroke care and sheds light on the fact that current procedural costs consume the bulk of hospitalization reimbursement, which may greatly affect the long-term sustainability of stroke centers.

\section{Conclusion}

The procedural costs of mechanical thrombectomy for AIS are increasing and account for the bulk of hospitalization reimbursement. The impact of these expenditures in the long-term sustainability of stroke centers deserves greater consideration. While is likely that the SR technology results in higher rates of optimal reperfusion, better clinical outcomes, and shorter LOS, larger studies are still needed to prove its cost-effectiveness.

\section{Disclosure Statement}

Raul G. Nogueira: Stryker Neurovascular (Trevo-2 and DAWN Trials Principal Instigator); Covidien (SWIFT and SWIFT Prime Trials Steering Committee; STAR Trial Angiographic Core Lab); Penumbra (3D Separator Trial Executive Committee); Rapid Medical (Stroke Trial DSMB). Rishi Gupta: Consultant: Stryker Neurovascular, Covidien, Rapid Medical; Steering Committee for Penumbra THERAPY trial; Associate Editor Journal of Neuroimaging; Associate Editor Interventional Neurology; royalties from UpToDate.

\section{References}

1 Miniño AM, Murphy SL, Xu J, Kochanek KD: National Vital Statistics Reports, vol. 59, No. 10. Hyattsville, National Center for Health Statistics, 2011.

-2 Demaerschalk BM, Hwang HM, Leung G: Cost analysis review of stroke centers, telestroke, and rt-PA. Am J Manag Care 2010;16:537-544.

3 Heidenreich PA, Trogdon JG, Khavjou OA, Butler J, Dracup K, Ezekowitz MD, et al: Forecasting the future of cardiovascular disease in the United States: a policy statement from the American Heart Association. Circulation 2011;123:933-944.

4 Hacke W, Donnan G, Fieschi C, Kaste M, von Kummer R, Broderick JP, et al: Association of outcome with early stroke treatment: pooled analysis of ATLANTIS, ECASS, and NINDS rt-PA stroke trials. Lancet 2004;363:768774.

5 Khatri P, Abruzzo T, Yeatts SD, et al; IMS I and II Investigators: Good clinical outcome after ischemic stroke with successful revascularization is time-dependent. Neurology 2009;73:1066-1072.

6 Sun CH, Nogueira RG, Glenn BA, Connelly K, Zimmermann S, Anda K, et al: 'Picture to puncture': a novel time metric to enhance outcomes in patients transferred for endovascular reperfusion in acute ischemic stroke. Circulation 2013;127:1139-1148.

7 Saver JL, Jahan R, Levy EI, Jovin TG, Baxter B, Nogueira RG, et al: Solitaire flow restoration device versus the Merci Retriever in patients with acute ischaemic stroke (SWIFT): a randomised, parallel-group, non-inferiority trial. Lancet 2012;380:1241-1249.

-8 Nogueira RG, Lutsep HL, Gupta R, Jovin TG, Albers GW, Walker GA, et al: Trevo versus Merci retrievers for thrombectomy revascularisation of large vessel occlusions in acute ischaemic stroke (TREVO 2): a randomised trial. Lancet 2012;380:1231-1240.

-9 Department of Health and Human Services. Centers for Medicare and Medicaid Services: Changes to the hospital inpatient prospective payment systems and fiscal year 2009 rates. Fed Regist 2008;73:48433-49084. http://www.access.gpo.gov/su_docs/fedreg/a080819c.html (accessed October 12, 2009). 
10 Taylor TN, Davis PH, Torner JC, Holmes J, Meyer JW, Jacobson MF: Lifetime cost of stroke in the United States. Stroke 1996;27:1459-1466.

11 Rha JH, Saver JL: The impact of recanalization on ischemic stroke outcome: a metaanalysis. Stroke 2007;38: 967-973.

12 Fagan SC, Morgenstern LB, Petitta A, Ward RE, Tilley BC, Marler JR, et al: Cost-effectiveness of tissue plasminogen activator for acute ischemic stroke. NINDS rt-PA Stroke Study Group. Neurology 1998;50:883-890.

-13 Saqqur M, Uchino K, Demchuk AM, Molina CA, Garami Z, Calleja S, et al: Site of arterial occlusion identified by transcranial Doppler predicts the response to intravenous thrombolysis for stroke. Stroke 2007;38:948-954.

14 Smith WS, Tsao JW, Billings ME, Johnston SC, Hemphill JC 3rd, Bonovich DC, et al: Prognostic significance of angiographically confirmed large vessel intracranial occlusion in patients presenting with acute brain ischemia. Neurocrit Care 2006;4:14-17.

$\rightarrow 15$ Nguyen-Huynh MN, Johnston SC: Is mechanical clot removal or disruption a cost-effective treatment for acute stroke? AJNR Am J Neuroradiol 2011;32:244-249.

16 Kim AS, et al: A cost utility analysis of mechanical thrombectomy as an adjunct to intravenous tissue plasminogen activator for acute large vessel ischemic stroke. Stroke 2011;42:2013-2018.

17 Smith WS, Sung G, Starkman S, Saver JL, Kidwell CS, Gobin YP, et al: Safety and efficacy of mechanical embolectomy in acute ischemic stroke: results of the MERCI trial. Stroke 2005;36:1432-1438.

18 Patil CG, Long EF, Lansberg MG: Cost-effectiveness analysis of mechanical thrombectomy in acute ischemic stroke. J Neurosurg 2009;110:508-513.

19 Turk AS 3rd, Campbell JM, Spiotta A, et al: An investigation of the cost and benefit of mechanical thrombectomy for endovascular treatment of acute ischemic stroke. J Neurointerv Surg 2014;6:77-80.

20 Spieler JF, Lanoë JL, Amarenco P: Costs of stroke care according to handicap levels and stroke subtypes. Cerebrovasc Dis 2004;17:134-142.

21 Chen M: Cost-effectiveness of endovascular therapy for acute ischemic stroke. Neurology 2012;79:S16-S21. 